\title{
Parametric Design and Rapid Prototyping of Installation Box for Vehicle Terminal PCB
}

\author{
Xingxing Wang ${ }^{1, \mathrm{a}}$, Hongjun $\mathrm{Ni}^{1, \mathrm{~b}}$, Yu Zhu ${ }^{1}$, Mingyu Huang ${ }^{1}$ and Jiawen Deng ${ }^{1}$ \\ ${ }^{1}$ School of Mechanical Engineering, Nantong University, 226019, Nantong, China
}

\begin{abstract}
Installation box for vehicle terminal PCB (Printed Circuit Board) was took as research object, which is encountered in the process of project developing. Vehicle terminal PCB in actual development process was set as an example, point cloud data were acquired by three coordinate measuring method; Imageware software was used to reconstruct the vehicle terminal PCB model, basic size parameters of vehicle terminal PCB can be got and then design parameters of installation box for vehicle terminal PCB can be determined. Design of the installation box for vehicle terminal PCB was completed based on Solidworks software, then 3D modeling and 2D drawing of installation box for vehicle terminal PCB was gained. Up Plus 2 rapid prototype machine was used to manufacture installation box for vehicle terminal PCB rapidly based on 3D printing technology, then prototype of installation box for vehicle terminal PCB was obtained. It is of certain engineering significant for single (small amount) manufacturing of installation box for general PCB.
\end{abstract}

Key words: Parametric Design, 3D Modeling, Rapid Prototyping, 3D Printing, Installation Box

\section{Introduction}

Parametric design is an important thought of Revit Building software, and it is divided into two parts: parametric graph and parameterized modifying engine. Graphics primitive in Revit Building are all appeared in the form of component. The difference between these components is reflected by the adjustment of the parameters, and the parameters preserved all information of graphics primitive which is digitalization construction component. Compared with the parametric design software which is wildly used, there are four type of application software can be the mainstream and they are Pro/Engineer, UGNX, CATIA and Solidworks. These four softwares have their own characteristics and have occupied a certain market share in different areas respectively [1]. RPM (Rapid Prototyping Manufacturing) manufacture the digital description model which includes the combined information of prototype (or parts, components) such as the geometry, material, structure and so on[2], and input these information into the mechanical and electrical control system which is controlled by computer to complete the "add" of material, and then finish the manufacturing of products[3].

According to the instance of vehicle terminal PCB which is encountered in the process of project developing, completed the parametric design and rapid prototyping of vehicle terminal PCB's installation box, with the aid of modern measuring technology and rapid prototyping technology.

\section{Obtain the point cloud date}

Digital measurement is also called data collection and it is the basis of reverse engineering. There are two main methods to obtain the point cloud, such as contact measuring and non-contact measuring. Contact measuring device has high measuring precision and strong adaptability, but its efficiency is low and can only measure the hard surface. Non-contact measuring device has high efficiency and high precision. Through the digital measurement, get a lot of point cloud data. The quality of the point cloud data directly affects the progress of the subject and the model reconstruction. Improving the accuracy of digital measurement is the precondition of the follow-up work smoothly.

Coordinate measuring machine (CMM) is a kind of contact measuring and is widely used to measure the three-dimensional sizes, forms, and positions of workpieces. In this part CMM is used and its equipment type is MQ8106. This machine's frame moving bridge structure is all closed. Its workbench is made of marble and its guideway is air-suspending. The measuring head block used in the measuring system is RENISHAW PH10T which can automatically twin twist to divide degree. The machine is equipped with RENISHAW needle group and HEADER F-Scan Flexible laser scanning probe, and has a model measuring function based on three-dimensional model. Not only do contacting measure, but also can do the non-contact laser scanning.

Before measuring the vehicle terminal PCB, needed

$\bar{a}$ wangxx@ntu.edu.cn, ${ }^{b}$ ni.hj@ntu.edu.cn (corresponding author)

(C) The Authors, published by EDP Sciences. This is an open access article distributed under the terms of the Creative Commons Attribution License 4.0 (http://creativecommons.org/licenses/by/4.0/). 
to open the air pump first. Then, connected the data line of coordinate measuring machine with the computer and start the computer. Put the manual controller in a particular place of coordinate measuring machine and be convenient to do the measuring work.

Measurement process done as follows: first of all, put the parts on the work surface and let it be fixed, and realized the shape measurement by manual controller. Second, Debugged coordinate measuring machine to make it back to zero, and then start measuring. After the completion of measurement, save the file with the format of "STL"[4], this can be used directly when dealing with the point cloud. Figure 1 shows the picture of vehicle terminal PCB measurement.

The date can be measured by coordinate measuring machine should be saved to be dealing with, and the format of the file data is still "STL". Because the vehicle terminal PCB parts are too small and complex, there were still some size cannot be measured, such as some holes and the relative position of two parts. As a result, it was necessary to use vernier caliper to complete the measurement.

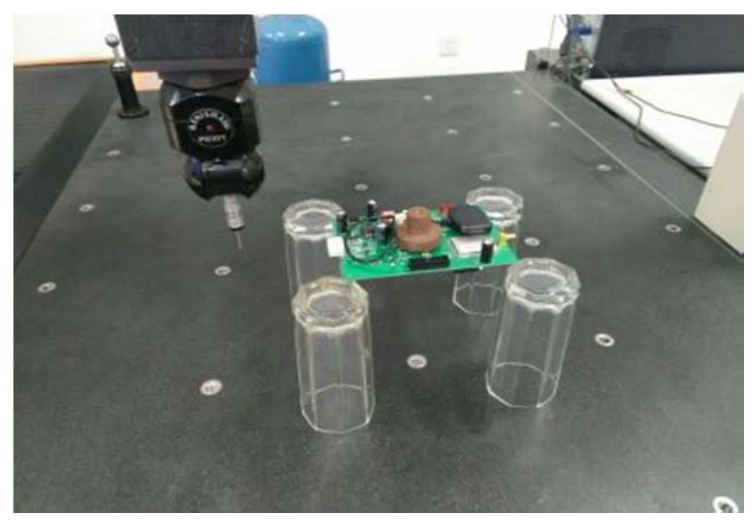

Figure 1. Measure of vehicle terminal PCB

\section{The point cloud processing and key parameter extraction of vehicle terminal PCB}

Imageware is a famous software in the field of the reverse engineering. Imageware software has strong processing capacity of point cloud, editing skills of surface and ability of surface construction. It is widely used in the area of automotive, aviation, aerospace, consumer appliances, mold design and manufacturing, computer parts, and so on [5].

Step 1: According to the three views in Imageware software, dealt with data. Put the point cloud data in the right place and corrected the sharp date. The reason for doing this was to make the point cloud data show more regulation in the software view, at the same time, it was convenient to correct the position and modify the data [6].

Step 2: Dealt with the point cloud data of vehicle terminal PCB (as shown in figure 2). Generated plane with the line existing, and made a mark on corresponding line and plane (as shown in figure 3). It was better to generate the corresponding entity in

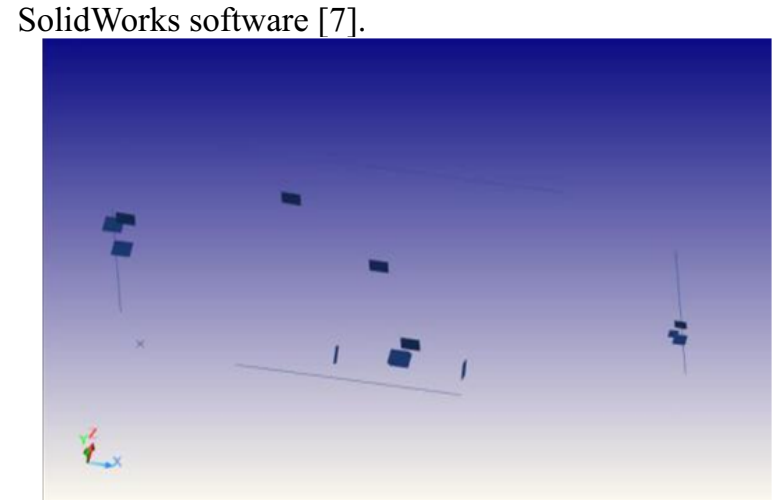

Figure 2. Point cloud data of vehicle terminal PCB

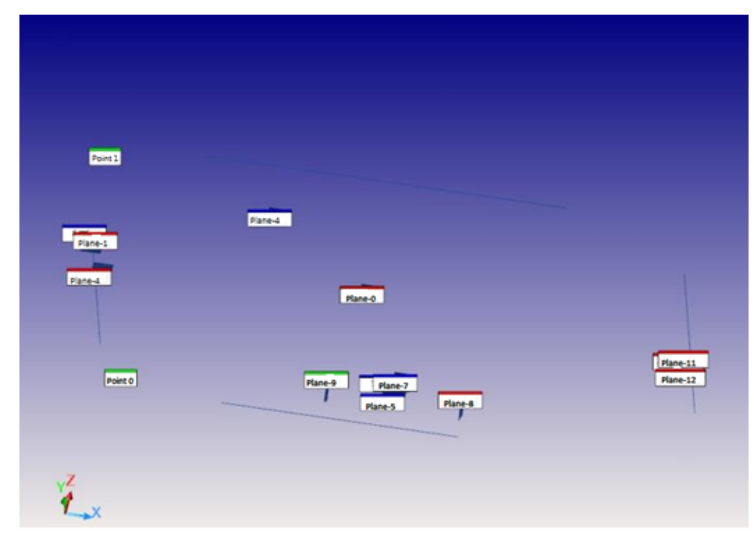

Figure 3. Relationship of line and plane of vehicle terminal PCB

It was necessary to get the information of key parameters of PCB, such as the size of the plane, the length of the line and the relationship of line and plane, in order to generate the corresponding 3D model better in the SolidWorks software. We sorted out the raw data and got the most direct parameters of vehicle terminal PCB. It was convenient to design the PCB install box based on the data. The date of line and plane of Vehicle terminal PCB is shown as table 1.

Table 1. Date of line and plane of vehicle terminal PCB

\begin{tabular}{|l|l|}
\hline project & parameter \\
\hline Length & $125 \mathrm{~mm}$ \\
\hline Distance from Plane 1 to plane 0 & $15.5331 \mathrm{~mm}$ \\
\hline Distance from right Plane 2 to line 2 & $46.6746 \mathrm{~mm}$ \\
\hline Distance from Plane 5 to line 3 & $29.7657 \mathrm{~mm}$ \\
\hline Distance from Plane 7 to plane 0 & $10.7061 \mathrm{~mm}$ \\
\hline Width & $89 \mathrm{~mm}$ \\
\hline Distance from left Plane 3 to line 0 & $46.2524 \mathrm{~mm}$ \\
\hline Distance from Plane 4 to plane 0 & $10.7450 \mathrm{~mm}$ \\
\hline Distance from Plane 6 to line 1 & $28.9435 \mathrm{~mm}$ \\
\hline Distance from Plane 8 to plane 0 & $9.6866 \mathrm{~mm}$ \\
\hline
\end{tabular}




\section{3D Modeling}

\subsection{Reconstruction of three-dimensional entity of vehicle terminal PCB.}

The file including the information of line and plane was saved as "Iges" format in Imageware Software. Opened the file in Solidworks software and dealt with the line and plane to reconstruct the model we want. Opened the file in SolidWorks and made a series of operations, such as stretching the matrix convex, extruded cut and so on, combined with the line and plane generated in Imageware, completed reconstructing the 3D model of vehicle terminal PCB

\subsection{Parametric design of vehicle terminal PCB's installation box.}

We had generated the 3D model of vehicle terminal PCB. Then, need do the parametric design of the installation box of vehicle terminal PCB according to the data obtained [8]. Because the card slot of vehicle terminal PCB was opening up, it was necessary to open a skylight, above the box so that connecting to the outside world. There was an antenna connection port, so it needed an antenna interface slot in the side of box. The slot to insert a card (SIM) was connected with the outside world, so also need to open a card slot. Taken other characteristics of the board into consideration, and the appearance of installation box's upper cover was generally come out. Design a skylight lid which was cooperates with the Skylight and they were connected with screw. The bottom of the box was also connected with the box cover with screw thread, and the bottom of the box was set inside the box cover.

Completed the design of every component of the installation box, and assembled all the parts and then got the 3D model with the 3D design software. To finish assembling the bottom and the box cover, let the hole in the bottom of the lid be concentric with the hole in the box cover, and matching the plane in convex with the plane in threaded column hole. This step assembled the installation box, and assembled the model of vehicle terminal PCB with the box as well, realized the virtual assembly.

Figure 4 shows the explosive view of final assembly of vehicle terminal PCB and installation box.

\section{Rapid Prototyping Manufacturing}

Rapid prototyping manufacturing, is also 3D printing (Three-Dimentional printing). It's working principle is that the hot melt materials which is processed into the sharp of filamentous (such as ABS), will be sent to hot nozzle through the wire feeding mechanism[9]. Then, filamentous material is heated and melted within the nozzle. At the same time, the nozzle moves along the outline of layer parts and extrudes the molten material, making it deposit and solidify in the specified position. The molten material which is solidified will be bonding with the previous layer which materials have already formed, and stacking layer upon layer repeatedly and eventually formed the product. This subject completed the manufacture of vehicle terminal PCB installation box with rapid prototyping machine (3D printer), which type is UP Plus 2[10].

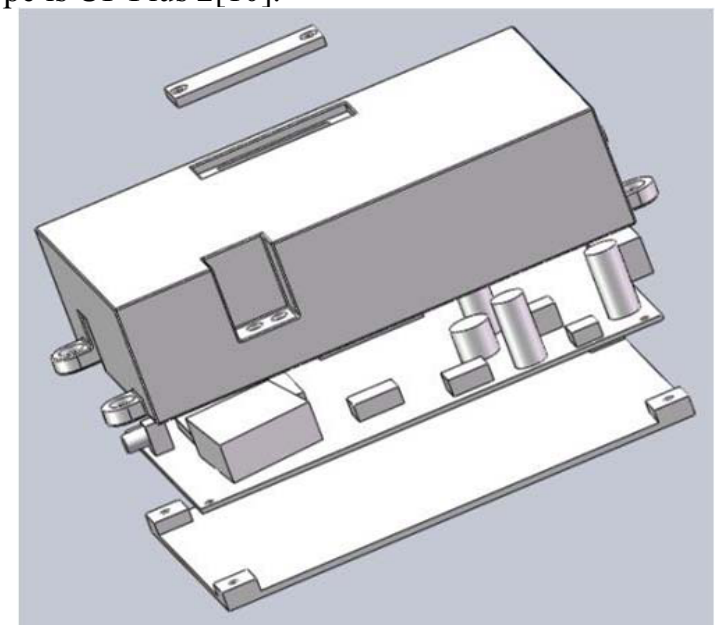

Figure 4. Explosive view of final assembly

Imported the STL file of vehicle terminal PCB's box cover into the computer. Because the size of installation box cover is larger than maximum working size of rapid prototyping machine, we divided the cover into two parts and then print it respectively. At the same time, the parameters were adjusted, such as the printing accuracy and the distance between nozzle and workbench. The printer can automatically print the product including support materials[11]. It took several hours during the printing process because the printer manufacture parts layer by layer.

There were four parts of the installation box: the left part of the box cover, the right part of the box cover, the bottom board, and the skylight lid, and they were all printed by the printer. Took the left part of the box cover as an instance. It took about 3 hours to complete the printing. Then got it out from the work plane of printer and done the post-processing work, such as removing the support material and polishing it slightly.

After printed all the components of the installation box, assembled them and got the box we want. Then, installed the Vehicle terminal PCB into the installation box. The result was fine, as shown in figure 5 .

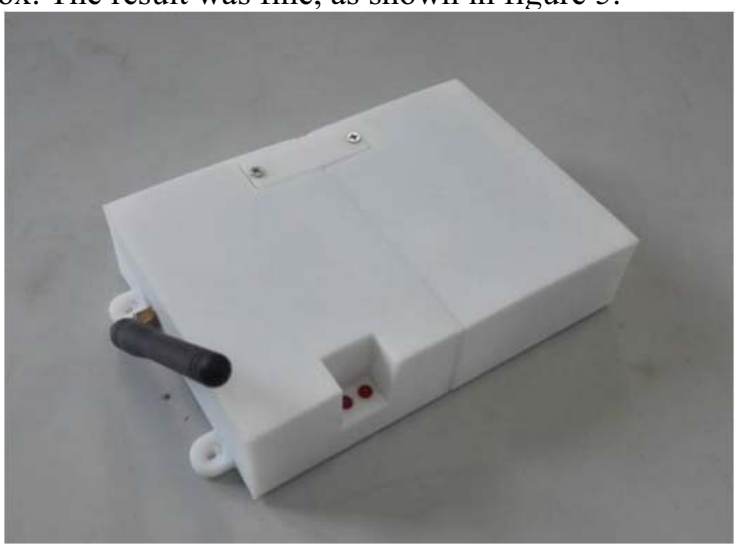

Figure 5. Vehicle terminal PCB installed 


\section{Summary}

1) Obtained the point cloud data of vehicle terminal $\mathrm{PCB}$, which was encountered in the process of project developing, by using coordinates measuring machine. Dealt with point cloud data of vehicle terminal PCB, extracted the basic size parameters, and determined the parameter requirements of designing vehicle terminal PCB installation box according to the Imageware software.

2) Completed the parametric design of vehicle terminal PCB based on Solidworks software, and finished the build of 3D model and 2D drawing of vehicle terminal PCB

3) With 3D printing technology, finished the manufacturing of vehicle terminal PCB installation box by $3 \mathrm{D}$ printer which type was UP Plus 2 , and got the product prototype of the installation box.

4) During the process of project development, this method will have certain value in engineering application for the rapid and economic manufacturing of one piece (small batch) PCB installation box.

\section{Acknowledgment}

This work was financially supported by National Science and Technology Support Program of China (2011BAG02B10), A Priority Academic Program Development of Jiangsu Higher Education Institutions (PAPD), Frontier and Key Technical Innovation Program of Nantong (MS22015028), and Nantong City
Applied Research Projects (BK2014053, GY12015028).

\section{References}

1. X.L. Li, K.C. Yu, X. Zhao. Manufacture Information Engineering of China. 39(13): 67-70 (2010)

2. H.J. Ni, L. Chen, X.B. Feng, et al. Mechanical design and manufacture (Chinese). 01: 137-139 (2014)

3. C. Weller, R. Kleer, F.T. Piller. Int. J. Prod. Econ. 164: 43-56 (2015)

4. H. Zhu, Z.F. Yang, W. Zhuang. Machine Tool and Hydraulics (Chinese). 37(6):186-189 (2009)

5. Y. Dan, B.F. Xie. The base of Imageware and reverse modeling technology (Tsinghua University Press, Beijing, 2006)

6. M. Vaezi, C. K. Chua. Int. J. Adv. Manuf. Tech. 52: 275-284 (2011)

7. P. Feng, X.M. Meng, J.F. Chen, et al. Constr. Build. Mater. 93: 486-497 (2015)

8. H.J. Ni, Y. Pei, S.S. Lv et al. Advanced Materials Research. 889-890: 14-21 (2014)

9. Y.Q. Li, T.C Shi. Machine Tool and Hydraulics, (Chinese), 38(23): 65-67 (2010)

10. B. Ezair, F. Massarwi, G. Elber. Comput. Graph. 51: 117-124 (2015)

11. M. Asadi-Eydivand, M. Solati-Hashjin, A. Farzad, et al. Robot Comput. Integr. Manuf. 37: 57-67 (2016) 